\title{
Development of Thai Silk Fibroin/Hyaluronic Acid Microspheres and the Application on Controlled Release of Curcumin
}

\author{
Piyarat Sungkhaphan $^{1, a}$, Juthamas Ratanavaraporn ${ }^{2}$, and Siriporn Damrongsakkul ${ }^{1, \mathrm{~b}, *}$ \\ 1 Department of Chemical Engineering, Faculty of Engineering, Chulalongkorn University, Bangkok 10330, \\ Thailand \\ 2 Biomedical Engineering Program, Faculty of Engineering, Chulalongkorn University, Bangkok 10330, \\ Thailand \\ E-mail: asungkhaphan.p@hotmail.com, bsiriporn.d@chula.ac.th (Corresponding author)
}

\begin{abstract}
The aim of this study was to develop microspheres from Thai silk fibroin (SF) blended with hyaluronic acid (HA) for controlled release application. HA was introduced to blend with SF to enhance properties of the microspheres. The SF-based microspheres could be prepared by water in oil emulsion and glutaraldehyde crosslinking technique. The obtained microspheres contained $71-91 \%$ SF and $9-29 \%$ HA. The microspheres having higher SF content showed more stability and slower degradation rate. Then, to prove the potential of SFHA microspheres as carriers for drug delivery, curcumin was absorbed in the microspheres. All microspheres released curcumin in a controllable manner, possibly due to the hydrophobic interaction between curcumin and crystalline domain of SF. Furthermore, it was shown that HA was self-released from the microspheres. The GAcrosslinked SFHA microspheres that simultaneously and controlled released curcumin and HA could be further developed and applied as injectable drug delivery system for treatment of diseases e.g. arthritis.
\end{abstract}

Keywords: Silk fibroin, hyaluronic acid, curcumin, microspheres, controlled release.

ENGINEERING JOURNAL Volume 21 Issue 4

Received 24 October 2016

Accepted 27 December 2016

Published 31 July 2017

Online at http://www.engj.org/

DOI:10.4186/ej.2017.21.4.139 


\section{Introduction}

Silk fibroin (SF) is a natural fibrous protein which is derived from domesticated silkworm, Bombyx mori. SF is composed of heavy $(\mathrm{H})$ and light $(\mathrm{L})$ chains linked by disulfide bond. A glycoprotein (p25) is also noncovalently linked to these chains. SF structure ( $\beta$-sheet of H-chain and amorphous of L-chain) contributes to its outstanding mechanical properties which offer an appropriate balance of modulus, strength and elongation. Other attractive properties of SF include biocompatibility, minimal inflammatory reaction, and slow biodegradation rate. Therefore, SF has recently been widely applied as scaffolding in tissue engineering such as vascular tube for flow diverting devices and stents [1-3], SF-composites used in skin, bone and cartilage [4-9]. Additionally, SF has been introduced as carriers for drug delivery system. SF and SFcomposited materials exhibit high encapsulation efficiency and controllable release kinetics, especially to hydrophobic molecules or drugs due to the hydrophobic interaction with crystalline region of SF [10-13].

Hyaluronic acid (HA) is a linear polysaccharide composed of $\mathrm{N}$-acetyl-D-glucosamine and glucoronic acid linked by $\beta-1,3$ and $\beta-1,4$ glucosidic linkages. Besides its biodegradability and biocompatibility, the remarked properties are viscoelasticity and ability to absorb large quantities of water $[14,15]$. Moreover, it regulates proliferation, migration, and differentiation of various cells $[16,17]$. HA has been extensively used for medical applications, including moisturizer to enhance wound healing, or a lubricant in eyes and joints of osteoarthritis disease $[18,19]$. HA has also been utilized as tissue engineered scaffolds and drug carriers $[19,20]$.

Curcumin is a substance of curcuminiods which is a polyphenolic compound. It is composed of two aromatic rings which have two groups of phenolic linked together by $\alpha, \beta$-unsaturated at the position of carbonyl groups, making the curcumin highly hydrophobic and water-insoluble. An antioxidant activity of curcumin by acting as a scavenger to unstable free radicals is widely reported [21, 22]. Curcumin can relief inflammation of the osteoarthritis and rheumatoid diseases by inhibition of reactive oxygen species (ROS) formed by activated macrophage [23-27]. Curcumin also shows anticancer activity by anti-angiogenesis and decreasing of the electrical potential of mitochondria membrane, resulting in the releasing of cytochrome-c and activating of some enzymes involved in cell death [28-30]. In addition, curcumin possesses other biological activities, for example, antiviral and antibacterial [21, 22, 31]. However, the high hydrophobicity, low stability, poor systemic bioavailability and rapid metabolism limit its therapeutic potential. Therefore, various curcumin delivery systems such as micro/nano particles, micelles, liposomes and scaffolds have been developed to enhance its bioavailability and stability [30-37]. Microparticle is an interesting choice of carrier in drug delivery system due to its high encapsulation efficiency as a result of large surface area per volume, well-defined model for degradation and drug release, controllable size, and can be applied as injectable carrier which is minimal invasive.

In this study, we have attempted to prepare microspheres from Thai silk fibroin (SF) blended with hyaluronic acid (HA) using water in oil emulsion and glutaraldehyde crosslinking technique. Based on our best knowledge, this is the first report of microspheres prepared from Thai SF protein blended with HA. The benefits of HA incorporation would be the enhanced biological activities and the modified degradation and drug release rate of the microspheres. We supposed that the developed SF/HA microspheres could release the hydrophobic curcumin in a controllable manner, resulting in the effectiveness of its biological action. Herein, the blending ratio of $\mathrm{SF} / \mathrm{HA}$ and crosslinking time were varied to modify morphology, degradation rate and stability of SF/HA microspheres. Then, curcumin was loaded into the SF/HA microspheres. The entrapment efficiency and in vitro release profile of curcumin from the microspheres were evaluated. The SF/HA microspheres with different composition and crosslinking extent were introduced as carrier for controlled delivery of curcumin.

\section{Materials and Methods}

\subsection{Materials}

The cocoons of B. mori Thai silkworm "Nangnoi Srisaket 1" were a kind gift from Queen Sirikit Sericulture Center, Srisaket province, Thailand. Hyaluronic acid (HA, $\bar{M}_{v} \sim 722 \mathrm{kDa}$, cosmetic grade) was purchased from Namsiang Co. Ltd., Thailand. Lithium bromide ( $\mathrm{LiBr})$, glutaraldehyde (GA), glycine, acetone and other chemicals used were analytical grade. 


\subsection{Preparation of Regenerated Thai SF Solution and HA Solution}

Regenerated Thai SF solution was prepared by subsequent processes of degumming and dissolution according to method previously reported [38]. Briefly, the cocoons were degummed by boiling in $0.02 \mathrm{M}$ sodium carbonate $\left(\mathrm{Na}_{2} \mathrm{CO}_{3}\right)$ twice to remove sericin and then rinsed thoroughly with deionized (DI) water, followed by drying at ambient temperature. To obtain aqueous SF solution, the degummed silk was dissolved in 9.3 M LiBr solution. The solution was dialyzed against DI water to remove neutral salts using dialysis tube $\left(\mathrm{MW}_{\text {cutoff }}=12,000-16,000\right)$ until the conductivity of dialyzed water was closed to that of DI water. The final concentration of obtained aqueous SF solution was around 6.5-7\%wt.

HA solution was prepared by direct dissolving in DI water and stirring overnight to ensure complete solubilization. Prior to the experiments, HA solution was sonicated at 40\% amplitude, 30\%cycle for 20 min to obtain less viscous HA solution. The average molecular weight of sonicated HA determined by intrinsic viscosity calculation using Huggins and Kramer equation was $260 \mathrm{kDa}$ [39].

\subsection{Preparation of SFHA Microspheres}

The SF/HA microspheres were fabricated by water in oil (W/O) emulsion technique. For an aqueous phase, $2.5 \%$ wt of $\mathrm{SF} / \mathrm{HA}$ aqueous solutions were prepared at $25^{\circ} \mathrm{C}, \mathrm{pH} 6$. For an oil phase, soy bean oil and Tween 80 were used as an oil and emulsifier, respectively. Then, the SF/HA mixture was added dropwise into oil phase (W/O ratio was 1/16) under a constant stirred speed of $300 \mathrm{rpm}, 25^{\circ} \mathrm{C}$ for $20 \mathrm{~min}$. Then, the emulsion temperature was decreased to $4^{\circ} \mathrm{C}$ and continuously stirred for $1 \mathrm{~h}$. The emulsion was stabilized by glutaraldehyde (GA) crosslinking. For this step, 3\%w/v GA was added dropwise into the emulsion. The crosslinking time was varied to 4,6 and $24 \mathrm{~h}$ under darkness condition. The obtained microspheres were washed repeatedly with acetone to remove excess oil and immersed in $0.1 \mathrm{M}$ glycine aqueous solution to remove unreacted aldehyde groups of GA, followed by rinsing with DI water. The GA-crosslinked microspheres were then freeze dried and size-fractionated using sieves $(32-85 \mu \mathrm{m})$.

The HA content in obtained SFHA microspheres was determined by modified Elson-Morgan assay [40, 41]. This was known to measure the amount of $\mathrm{N}$-acetyl-D-glucosamine in HA molecules. Briefly, $10 \mathrm{mg}$ microspheres were hydrolyzed with hydrochloric acid $(\mathrm{HCl})$ at $96^{\circ} \mathrm{C}$ for $14 \mathrm{~h}$, then the $\mathrm{pH}$ of hydrolyzed solution was adjusted to 7 with $4 \mathrm{~N}$ of sodium hydroxide $(\mathrm{NaOH})$. Acetyl acetone solution was prepared by mixing $25 \mathrm{ml}$ of $1.25 \mathrm{~N}$ sodium carbonate $\left(\mathrm{Na}_{2} \mathrm{CO}_{3}\right)$ with $0.75 \mathrm{ml}$ of acetyl acetone reagent. Then, $2 \mathrm{ml}$ of acetyl acetone solution was mixed with $2 \mathrm{ml}$ of hydrolyzed solution and further incubated at $96^{\circ} \mathrm{C}$. Ehrlich's regent was prepared by mixing p-dimethylaminobenzaldehyde (DMAB), $6 \mathrm{~N} \mathrm{HCl}$ and 95\%vol ethanol together. Then, $2 \mathrm{ml}$ of Ehrlich's regent was added into the obtained solution. The absorbance of the solution was spectrophotometrically measured at the wavelength of $528 \mathrm{~nm}$. The amount of HA was determined from a standard curve of $\mathrm{N}$-acetyl-D-glucosamine. The amount of HA in microspheres was calculated in relative to the calculated amount of HA in the preparation process as follows:

$$
\frac{W_{G l c N A c}}{M W_{G I c N A c}}=\frac{W_{H A}}{M W_{H A}}
$$

where $W_{G l c N A c}$ and $W_{H A}$ represent the weight of measured $\mathrm{N}$-acetyl-D-glucosamine in microspheres and calculated weight of HA in the microsphere preparation, respectively. While $M W_{G l c N A c}$ and $M W_{H A}$ represent the molecular weight of repeating unit of $\mathrm{N}$-acetyl-D-glucosamine and HA, respectively.

\subsection{Morphology and Size Analysis of Microspheres}

The SF/HA microspheres were immersed in DI water for saturated swelling and dried under critical temperature and critical pressure. The morphology of dried SFHA microspheres was observed on a scanning electron microscope (SEM, JSM-5410LV, JEOL Ltd, Japan) at the accelerating voltage of 12-15 $\mathrm{kV}$ after sputter-coating with gold. The SF/HA microspheres were randomly cut to observe crosssectioned morphology. The average size of SF/HA microspheres $(5 \mathrm{mg})$ was determined by Mastersizer 2000 (Malvern Instrument Ltd.) (n=3). 


\subsection{Stability Test of SF/HA Microspheres}

The stability of SF/HA microspheres was evaluated after immersing microspheres in DI water at $37^{\circ} \mathrm{C}$ for different time intervals. The amounts of HA in the supernatant were determined by modified ElsonMorgan assay as described earlier. The remained microspheres were freeze dried and weighed. The percentage of weight loss was calculated as follows:

$$
\text { Weight loss }(\%)=\frac{W_{i}-W_{f}}{W_{i}} \times 100
$$

where $W_{i}$ and $W_{f}$ represent dried weight of $\mathrm{SF} / \mathrm{HA}$ microspheres before and after incubated with DI water, respectively $(\mathrm{n}=3)$.

\subsection{In vitro Biodegradation Test}

$\mathrm{SF} / \mathrm{HA}$ microspheres were incubated in $1 \mathrm{U} / \mathrm{ml}$ of Protease XIV solution (pH 7.4) containing $0.01 \% \mathrm{wt}$ sodium azide as anti-bacterial agent at $37^{\circ} \mathrm{C}$. The enzymatic solution was changed every 2 days. The remained microspheres after incubation were washed with DI water and freeze dried. The dried microspheres were weighed and the percentage of remaining weight at different time intervals was calculated as follows:

$$
\text { Remaining weight }(\%)=\frac{W_{a}}{W_{b}} \times 100
$$

where $W_{b}$ and $W_{a}$ represent weight of $\mathrm{SF} / \mathrm{HA}$ microspheres before and after enzymatic incubation at different time intervals, respectively $(\mathrm{n}=3)$.

\subsection{Evaluation of Entrapment Efficiency and Loading of Curcumin in the SF/HA Microspheres}

Curcumin loading in microspheres were carried out by absorption method as follows. Curcumin solution (5 $\mathrm{mg} / \mathrm{ml}$ in absolute ethanol) was absorbed on $5 \mathrm{mg}$ of SF/HA microspheres. The absorbed microspheres were left at $4^{\circ} \mathrm{C}$ overnight and air dried for $24 \mathrm{~h}$, then the curcumin loaded SF/HA microspheres were obtained. The entrapment efficiency and drug loading were determined by repeatedly washing curcumin loaded SF/HA microspheres with dimethyl sulfoxide (DMSO). The absorbance of curcumin eluted in DMSO was measured by a spectrophotometer at the wavelength of $430 \mathrm{~nm}[42,43]$. The entrapment efficiency and drug loading were calculated as follows:

$$
\begin{aligned}
& \text { Entrapment efficiency }=\frac{M_{a}}{M_{t}} \times 100 \\
& \text { Drug loading }(\%)=\frac{M_{a}}{M_{a}+M_{s}} \times 100
\end{aligned}
$$

where $M_{a}$ and $M_{t}$ represent actual and theoretical weight of curcumin in SF/HA microspheres, respectively. $M_{s}$ represents the weight of SF/HA microspheres (n=3).

\subsection{In vitro Release of Curcumin from SF/HA Microspheres}

The in vitro release of curcumin was evaluated in enzymatic condition using protease XIV. The known amount of curcumin loaded SF/HA microspheres were dispersed in $10 \mathrm{ml}$ phosphate buffer saline (PBS, $\mathrm{pH}$ 7.4) containing $10 \% \mathrm{vol}$ fetal bovine serum (FBS) and $1 \mathrm{U} / \mathrm{ml}$ of protease XIV and incubated at $37^{\circ} \mathrm{C}$. At predetermined time intervals, the supernatant was collected and the absorbance of curcumin in releasing medium was measured using a spectrophotometer at the wavelength of $430 \mathrm{~nm}$. The fresh releasing medium was replaced with the same volume. The concentration of released curcumin was calculated from a standard curve of curcumin prepared in phosphate buffer saline (PBS) at various concentrations. The percentage of cumulative release of curcumin from SF/HA microspheres was calculated as follows: 


$$
\begin{gathered}
\qquad C_{i}=\sum_{i=0}^{t} M_{i} C_{i} \\
\text { Cumulative release (\%) }=\frac{C_{i}}{C_{T}} \times 100
\end{gathered}
$$

where $C_{i}, M_{i}$ and $C_{T}$ represent the amounts of released curcumin from $\mathrm{SF} / \mathrm{HA}$ microspheres at time intervals, released curcumin from SF/HA microspheres at time $i$ and total amount of curcumin entrapped in microspheres $(\mathrm{n}=3)$.

\subsection{Mathematical Modelling of Curcumin Release}

To explain the kinetic and mechanism of curcumin release, the release profile data were fitted into a Power law model. The calculation was determined as follows:

$$
\frac{M_{t}}{M_{\infty}}=k t^{n}
$$

where $\frac{M_{t}}{M_{\infty}}$ represents the fraction of curcumin release at time t, and $k, n$ represent release constant and release exponent, respectively. For the power law model (Spherical system), a value of $n=0.43$ indicates a fickian diffusion mechanism, a value of $\mathrm{n}=0.85$ indicates a case II transport (relaxation or erosion mechanism) and a value of $\mathrm{n}$ between 0.43 and 0.85 indicates an anomalous transport [44-46].

\subsection{Statistical Analysis}

One way analysis of variance (ANOVA) was performed to compare individual set of data. In all cases, the results were considered to be significantly different when $p$-value $<0.05(\mathrm{n}=3)$.

\section{Results}

\subsection{HA Content in SF/HA Microspheres}

The percentage of HA presented in GA-crosslinked SF/HA microspheres is shown in Fig. 1. The amounts of HA in 3 formulations of GA-crosslinked microspheres were about $18-29 \%, 11-24 \%$ and $9-14 \%$, respectively. The microspheres were then named based on the actual percentage of $\mathrm{HA}$ as follows: SF82HA18, SF89HA11, and SF91HA9. Considering the effect of crosslinking time, it was observed that the percentage of HA in the GA-crosslinked microspheres seemed to be increased with the increasing crosslinking time. 


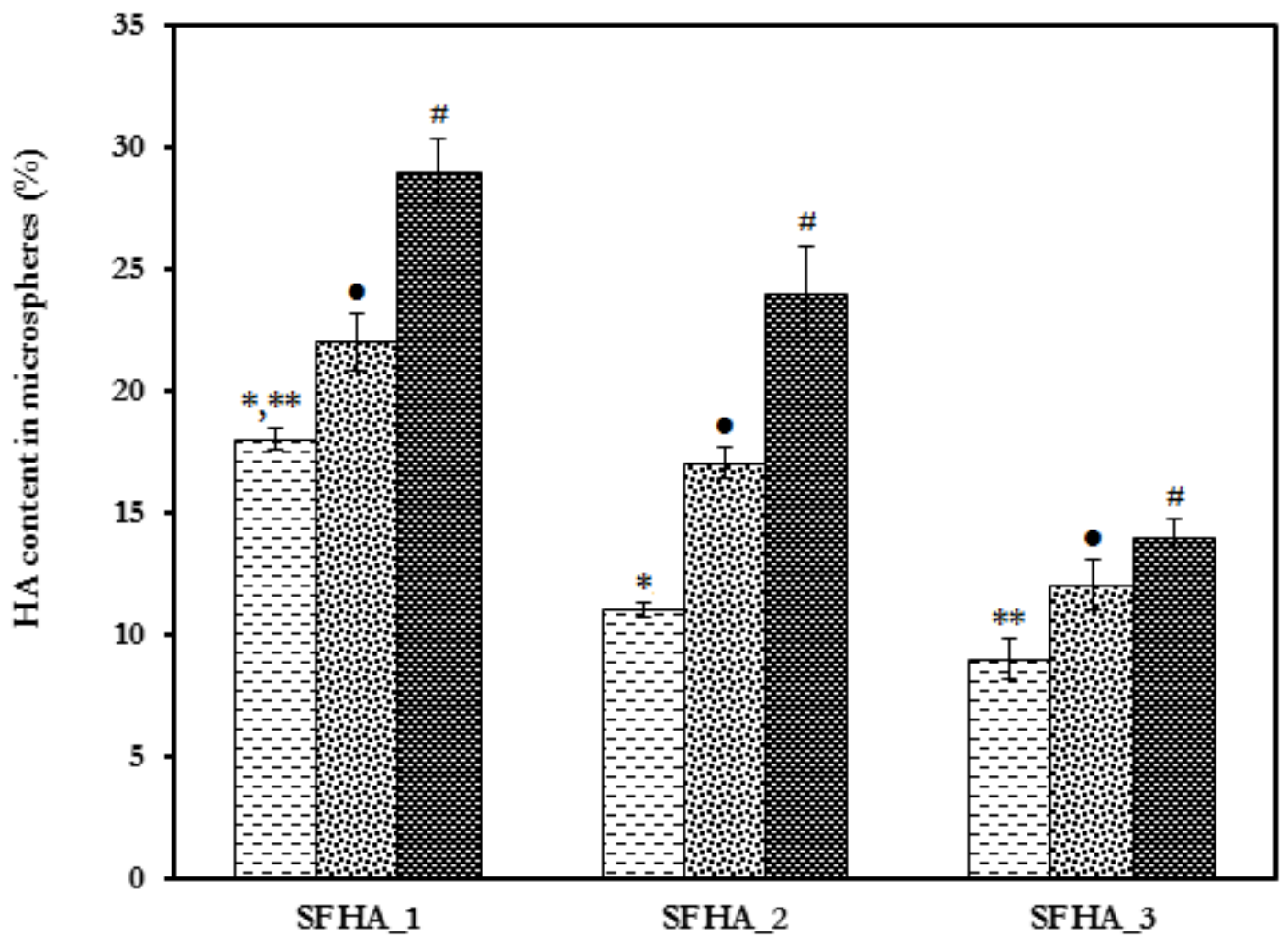

Fig. 1. Percentage of HA presented in GA-crosslinked SFHA microspheres crosslinked for 4(可), 6(圈), and $24 \mathrm{~h}(\text { 圈 })^{*}, * *, \bullet, \# p<0.05$ when comparing among sample groups at corresponding crosslinking time.

\subsection{Morphology, Size and Yield of SF/HA Microspheres}

Surface morphology, average size, and yield of the dried GA-crosslinked SF/HA microspheres are presented in Table 1. The SF/HA microspheres prepared at different weight blending ratios and crosslinked for $6 \mathrm{~h}$ were spherical with smooth surface. Some aggregated and irregular shaped particles were observed on microspheres crosslinked with GA for 4 and $24 \mathrm{~h}$. The cross-sectioned morphology of the microspheres revealed a non-porous inner structure (data not shown). Sizes and yields of the microspheres after fractionated were around 34-68 $\mu \mathrm{m}$ and 51-67\%, respectively. It was noticed that the size of the microspheres tended to be bigger for the microspheres containing higher SF content.

Table 1. Morphology, sizes and yields of GA-crosslinked SFHA microspheres prepared at different compositions and crosslinked for 4, 6 and $24 \mathrm{~h}$ (scale bar $=10 \mu \mathrm{m})$.

\begin{tabular}{|c|c|c|c|}
\hline $\begin{array}{l}\text { Crosslinking time } \\
\text { (h) }\end{array}$ & SEM images & $\begin{array}{l}\text { Size of microspheres } \\
(\mu \mathrm{m})\end{array}$ & $\begin{array}{l}\text { Yield of microspheres } \\
(\%)\end{array}$ \\
\hline \multirow{2}{*}{4} & SF82HA18 & $57.6 \pm 3.1$ & $51.8 \pm 1.9$ \\
\hline & SF89HA11 & $57.0 \pm 2.3$ & $56.5 \pm 5.9$ \\
\hline
\end{tabular}




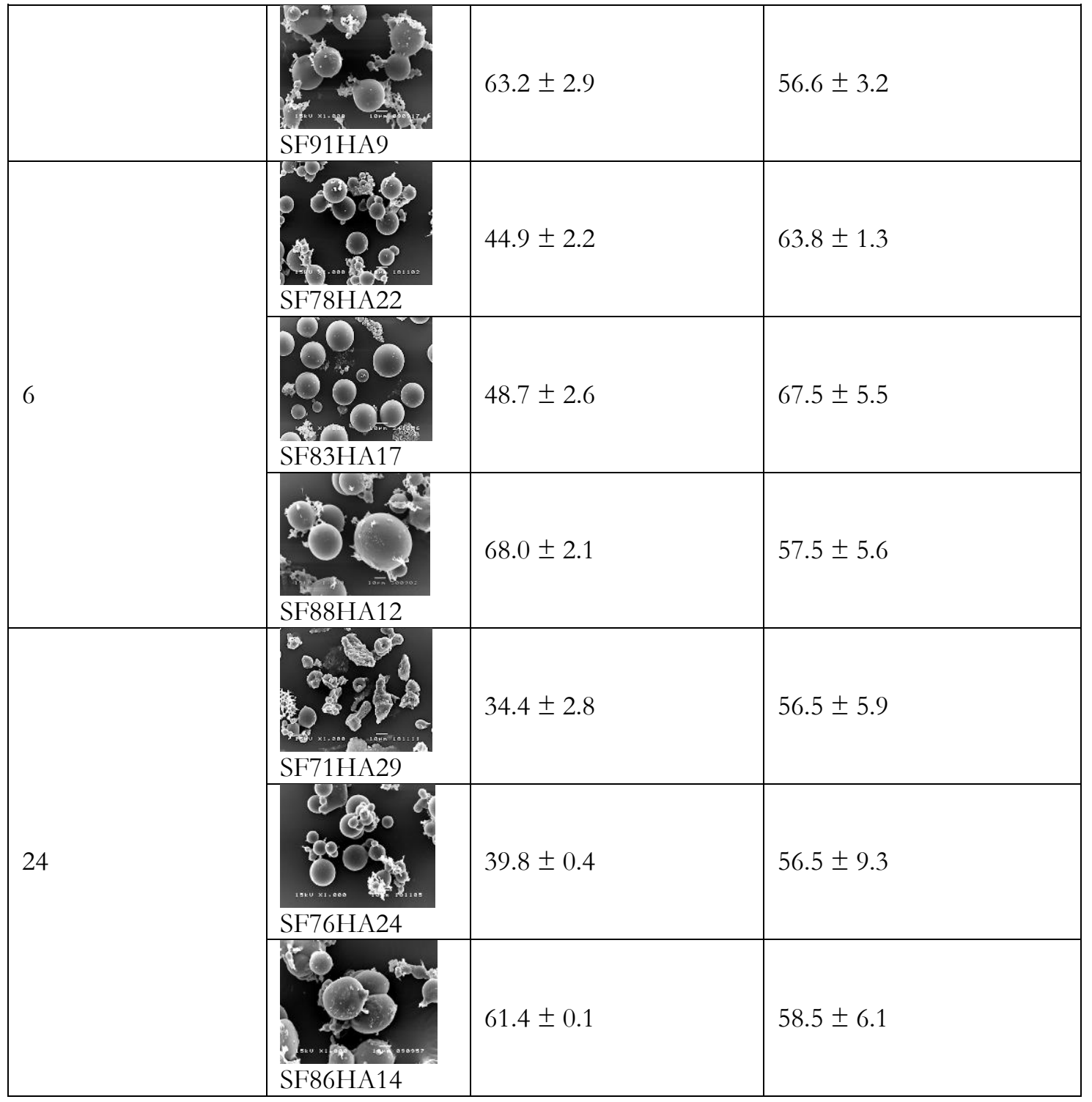

\subsection{Stability of SF/HA Microspheres}

The amount and percentage of $\mathrm{HA}$ loss from SF/HA microspheres after immersion in DI water are demonstrated in Fig. 2. The weight loss of HA per gram of freeze-dried microspheres continuously increased with time. The microspheres having higher HA content showed a higher amount of HA loss. The percentages of HA loss of all microspheres were around $7-21 \%$ after immersion for $144 \mathrm{~h}$. The microspheres crosslinked for $4 \mathrm{~h}$ revealed higher percentage of HA loss when comparing to corresponding microspheres crosslinked for 6 and $24 \mathrm{~h}$. Meanwhile, the amounts of HA detected in supernatant of GAcrosslinked SF/HA microspheres after immersion in DI water are shown in Fig. 3. The amount of HA in the supernatant of microspheres having higher HA content was noticed to be higher than that found in the supernatant of microspheres containing lower HA content. It was also observed that the amount of HA in supernatant was decreased in the microspheres crosslinked for longer time. The highest amount of HA $(0.07-0.20 \mathrm{mg} / \mathrm{g}$ microspheres) was detected in the supernatant of the microspheres crosslinked for $4 \mathrm{~h}$. 


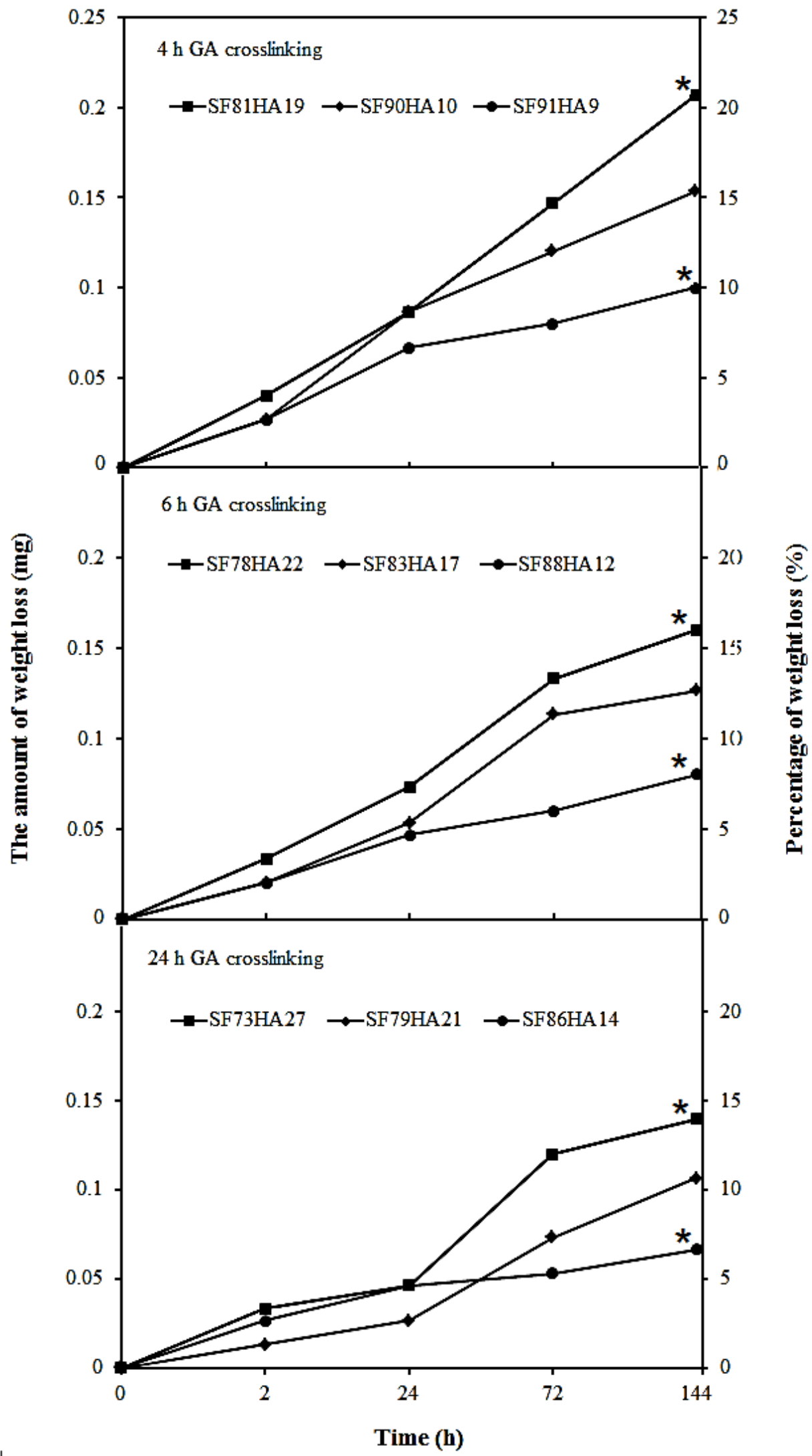

Fig. 2. The amount of weight loss of GA-crosslinked SFHA microspheres (left) and the percentage of weight loss (right) after immersion in DI water at $37^{\circ} \mathrm{C}$ for different time intervals. ${ }^{*} p<0.05$ when comparing among sample groups at corresponding time. 


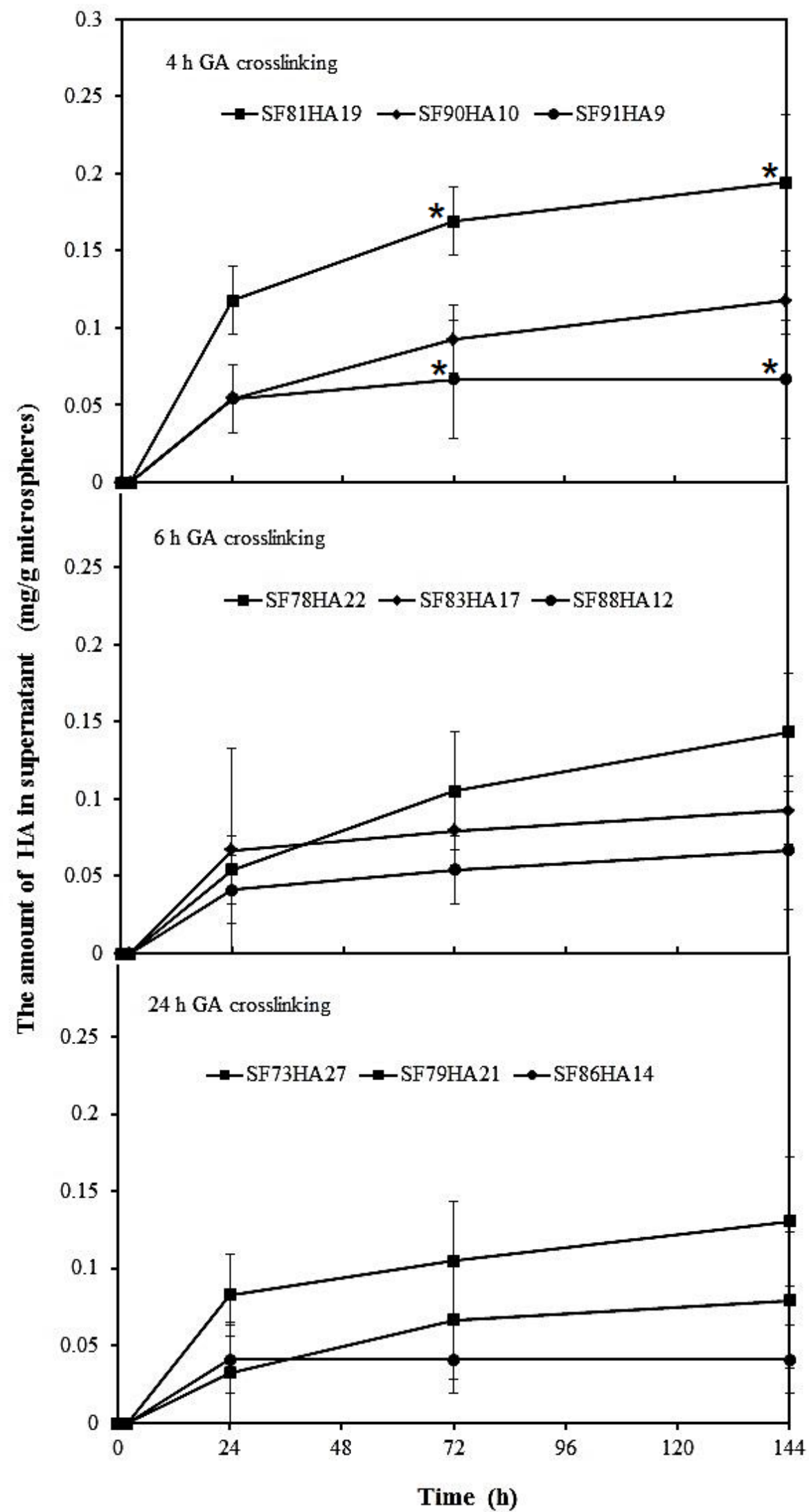

Fig. 3. The amount of HA detected in supernatant after immersed the GA-crosslinked SFHA microspheres in DI water at $37^{\circ} \mathrm{C}$ for different time intervals. ${ }^{*} p<0.05$ when comparing among sample groups at corresponding time. 


\subsection{In vitro Biodegradation of SF/HA Microspheres}

Figure 4 shows the degradation profiles of SF/HA microspheres crosslinked for $6 \mathrm{~h}$ after incubated in 1 $\mathrm{U} / \mathrm{ml}$ of protease $\mathrm{XIV}$ at $37^{\circ} \mathrm{C}$ for a period of 14 days. The microspheres containing higher SF content show slightly higher percentage of weight remaining than those having lower SF content. After 14 days of incubation, the weight of microspheres having the highest SF content (SF88HA12) remained 50\% while those having the lowest SF content (SF78HA22) were only 35\% of their original weights.

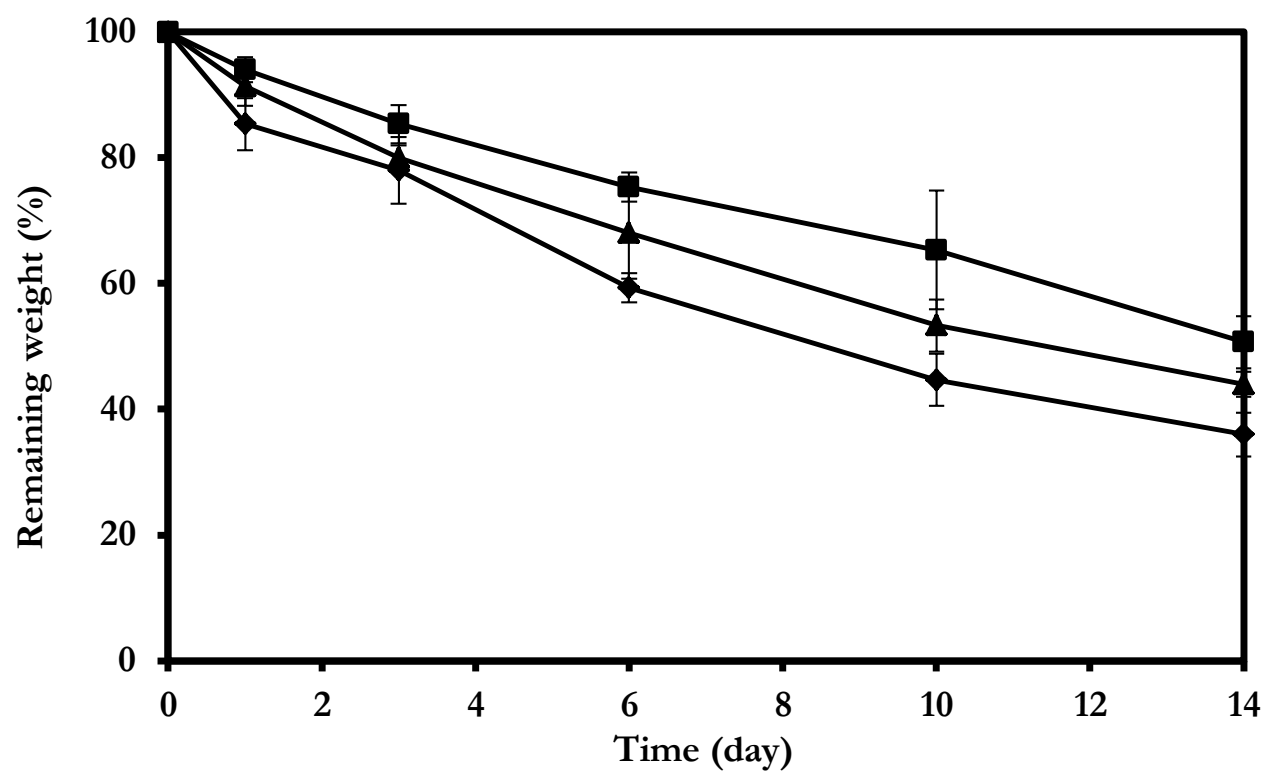

Fig. 4. Degradation profile of SFHA microspheres crosslinked with GA for $6 \mathrm{~h}$ after incubated in PBS solution ( $\mathrm{pH} 7.4$ ) containing $10 \% \mathrm{vol} F B S$ and $1 \mathrm{U} / \mathrm{ml}$ of protease XIV at $37^{\circ} \mathrm{C}$ for different time intervals.

SF88HA12; ( $\mathbf{\Delta})$ SF83HA17; ( ) SF78HA22.

\subsection{Entrapment and Loading Efficiency of Curcumin in SF/HA Microspheres}

Table 2 demonstrates the entrapment and loading efficiency of curcumin in SF/HA microspheres. The significant difference in the entrapment and loading efficiencies of curcumin in various formulations of SF/HA microspheres was not observed. The entrapment and loading efficiencies of curcumin in all microspheres crosslinked with GA for $6 \mathrm{~h}$ were approximately $30-33 \%$ and $7.1-7.7 \%$, respectively.

Table 2. Entrapment and loading efficiency of curcumin in SFHA microspheres crosslinked with GA for $6 \mathrm{~h}$.

\begin{tabular}{lll}
\hline Sample & Entrapment efficiency (\%) & Loading efficiency (\%) \\
\hline SF78HA22 & $33.2 \pm 1.1$ & $7.7 \pm 0.2$ \\
\hline SF83HA17 & $32.7 \pm 5.3$ & $7.5 \pm 1.1$ \\
\hline SF88HA12 & $30.8 \pm 1.7$ & $7.1 \pm 0.4$ \\
\hline
\end{tabular}

\subsection{In vitro Release of Curcumin from SF/HA Microspheres}

The profiles of curcumin release from SF/HA microspheres crosslinked with GA for $6 \mathrm{~h}$ after incubated in PBS solution ( $\mathrm{pH} 7.4$ ) containing $10 \% \mathrm{v}$ FBS and $1 \mathrm{U} / \mathrm{ml}$ of protease XIV are shown in Fig. 5. Initially, the curcumin was continuously released to around $80 \%$ of original loaded amount. The release of curcumin became steady after $36 \mathrm{~h}$ (79-84\% of curcumin released). The significant difference in curcumin release rate among various microspheres was not observed. 


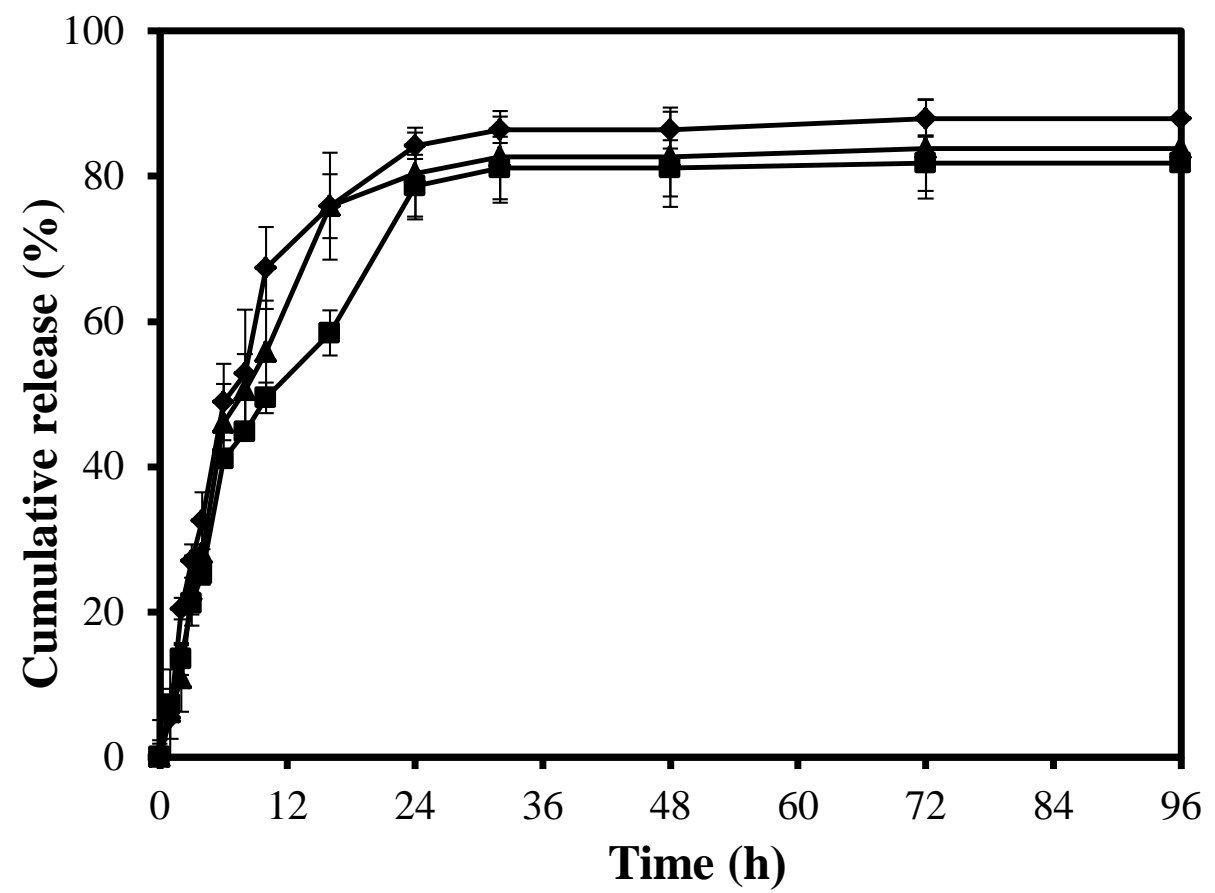

Fig. 5. In vitro release profile of curcumin from SFHA microspheres crosslinked with GA for $6 \mathrm{~h}$ after incubated in PBS solution ( $\mathrm{pH}$ 7.4) containing $10 \% \mathrm{vol} F B S$ and $1 \mathrm{U} / \mathrm{ml}$ of protease XIV at $37^{\circ} \mathrm{C}$ for different time intervals. ( $)$ SF88HA12; ( $)$ SF83HA17; ( ) SF78HA22.

Table 3. The constant and exponential values of curcumin released from SFHA microspheres crosslinked with GA for $6 \mathrm{~h}$ when fit to a power law model.

\begin{tabular}{llll}
\hline Sample & $\begin{array}{l}\text { Release } \\
\text { constant (k) }\end{array}$ & $\begin{array}{l}\text { Release } \\
\text { exponent (n) }\end{array}$ & $\begin{array}{l}\text { Regression } \\
\text { coefficient }\left(\mathbf{R}^{2}\right)\end{array}$ \\
\hline SF78HA22 & $0.16 \pm 0.09$ & $0.51 \pm 0.08$ & 0.87 \\
SF83HA17 & $0.14 \pm 0.09$ & $0.55 \pm 0.07$ & 0.91 \\
SF88HA12 & $0.14 \pm 0.07$ & $0.52 \pm 0.06$ & 0.85 \\
\hline
\end{tabular}

\section{Discussion}

In this study, we have developed microspheres from Thai SF and HA to be applied as a carrier of curcumin for controlled release applications. The SF/HA microspheres were successfully fabricated by a simple water in oil emulsion technique using Tween 80 as an emulsifier. GA was used to crosslink SF/HA microspheres during the microdroplet formation. In this case, aldehyde groups of GA would activate hydroxyl groups of HA and amino groups of SF to form intermolecular covalent bonds. Then, the GA-crosslinked SF/HA microspheres were obtained. The effects of crosslinking time and blending ratios of SF and HA on morphology, size, yield, and stability of SF/HA microspheres were elucidated.

The SEM micrographs revealed a round shape with smooth surface of the microspheres crosslinked for $6 \mathrm{~h}$ while some aggregated and irregular shaped particles were found in microspheres crosslinked for 4 and $24 \mathrm{~h}$ (Table 1). This might imply that both 4 and $24 \mathrm{~h}$ were not appropriate time for the crosslinking reaction to occur. The $4 \mathrm{~h}$ crosslinking may be too short to achieve the complete crosslinking, resulting in unstable microspheres which became aggregated. And there were some high contents of uncrosslinked molecules were also leached out during microspheres formation. (the results shown in Figs. 2 and 3) On the other hand, the irregular shaped particles were obtained from the microspheres crosslinked for $24 \mathrm{~h}$, possibly being a result of mechanical damage from continuous stirring during crosslinking. Then, $6 \mathrm{~h}$ crosslinking time was suitable for microspheres preparation. The crosslinking time also influence on the incorporation of $\mathrm{HA}$ in SF/HA microspheres. More HA contents could be stabilized in SF/HA microspheres when applying a longer crosslinking time (Fig. 1). Furthermore, the sizes of the obtained microspheres after fractionation $(34-68 \mu \mathrm{m})$ were in the appropriate range for injection through a medical 
needle which is considered as a minimal invasive treatment [47, 48]. The stability of GA-crosslinked $\mathrm{SF} / \mathrm{HA}$ microspheres depended on both microsphere composition and crosslinking time. The lower stability of microspheres having higher HA content (Figs. 2 and 3) was explained by the structural characteristic of HA and SF as well as the GA crosslinking ability. HA is an anionic and non-sulfated polysaccharide which is hydrophilic and water-soluble. The dissolution of HA could then be easily occurred. On the other hand, SF molecules are known to be quite stable and water insoluble due to their hydrophobicity and secondary structure. Considering GA crosslinking reaction, GA could crosslink only at hydroxyl groups of HA molecules and it required two groups of hydroxyl to complete the crosslinking. Moreover, the H-O bond energy in hydroxyl group of HA was higher than $\mathrm{H}-\mathrm{N}$ bond energy in amino group of SF [49]. Therefore, the HA may have less ability to crosslink within the microspheres by GA reaction, resulting in its less stability. For further investigation of this study, all SF/HA microspheres crosslinked with GA for $6 \mathrm{~h}$ were selected because of their controlled morphology without agglomeration, appropriate size, yield, and stability.

We showed that the SF/HA microspheres crosslinked with GA for $6 \mathrm{~h}$ were enzymatically degraded by Protease XIV [50-52]. Protease XIV specifically cleaves the peptide bonds in SF molecules. The degradation profiles confirmed that large amount of SF in SF/HA microspheres was proteolytic degraded by protease XIV (Fig. 4). Meanwhile, HA as a water soluble molecule would be either hydrolyzed or degraded by enzyme. This explained the faster degradation rate of the microspheres containing higher HA content.

To investigate the potential of SF/HA microspheres as carriers for drug delivery, curcumin was used as a model drug. After curcumin was loaded in the microspheres, the entrapment and loading efficiencies were evaluated. The significant difference in entrapment and loading efficiencies of curcumin among different microspheres were not observed in this study, possibly because all microsphere formulations were SF-based (SF 78-88\%). The entrapment mechanism of curcumin in our SF-based microspheres might be explained by the hydrophobic interaction between the hydrophobic molecules of curcumin and the hydrophobic crystalline domain of SF [37, 53].

The release study under in vitro enzymatic condition revealed the controllable release profiles of curcumin from all microspheres (Fig. 5). The release of curcumin was fast at initial period due to the diffusion mechanism. Afterwards, the curcumin would be sustained released along the degradation of microspheres. Therefore, there may be two main mechanisms that regulate the release behavior of curucmin, diffusion and carrier's degradation [38, 54]. Comparing among different microsphere formulations, the release rate of curcumin was not significantly different. However, it seemed that the microspheres having higher SF content showed a slightly slower rate of curcumin release. This may also be explained by the hydrophobic interaction between curcumin and SF [10-13, 37, 53]. Ratanavaraporn et al. have reported the controlled release of curcumin from different compositions of gelatin/SF microspheres. It was found that the presence of SF in microspheres could prolong curcumin release [53]. To explain the mechanism of curcumin release from the microspheres, the release data were fit to a mathematical model based on regression analysis. The release kinetics and mechanisms of curcumin could be described by a power law model. The values of release exponent (n) of curcumin released from all microspheres were between 0.43 to 0.85 (Table 3), indicating that the mechanism controlling curcumin release were both diffusion and swelling or erosion (anomalous transport). It was supposed that the absorbed curcumin on the microspheres' surface was initially released out by diffusion-controlled mechanism. Thereafter, the curcumin attached on the crosslinked microspheres, especially on the hydrophobic domain of SF, was released out by microspheres' dissolution and degradation-controlled mechanism. In addition to the curcumin, HA was also continuously self-released from the microspheres due to its dissolution property (Fig. 4). Taken together, the GA-crosslinked SF/HA microspheres that simultaneously and sustained released curcumin and HA were suggested to be applied as injectable drug delivery system for the treatment of diseases e.g. arthritis. However, the further development of SF/HA microspheres is needed to achieve the carriers with extended range of sustained release of both curcumin and HA.

\section{Conclusion}

The SF/HA microspheres were successfully prepared by water-in-oil emulsion and glutaraldehyde crosslinking techniques. The obtained microspheres were SF-based. Blending composition of SF and HA and crosslinking time influenced on morphology, size, stability, and degradation rate of the microspheres. The microspheres containing higher SF content were slightly bigger and more stable while the 
microspheres crosslinked for $6 \mathrm{~h}$ appeared in a spherical shape with smooth surface due to an optimal crosslinking time. All microspheres could entrap curcumin as a hydrophobic molecule at around 30-33\% and released it in a controllable manner, possibly due to the hydrophobic interaction between curcumin and hydrophobic domain of SF. These SF/HA microspheres could be further developed to be applied as injectable carriers for the treatment of diseases e.g. arthritis.

\section{Acknowledgements}

This work has been supported by Chulalongkorn Academic Advancement into its 2nd Century Project (CUAASC).

\section{References}

[1] F. Causin, R. Pascarella, G. Pavesi, R. Marasco, G. Zambon, R. Battaglia, and M. Munari, "Acute endovascular treatment (b48 hours) of uncoilable ruptured aneurysms at non-branching sites using silk flow-diverting devices," M. Interv. Neuroradiol, vol. 17, no. 3, pp. 357-364, 2011.

[2] M. Leonardi, L. Cirillo, F. Toni, M. Dall'Olio, C. Princiotta, A. Stafa, L. Simonetti, and R. Agati, "Treatment of intracranial aneurysms using flow-diverting silk stents (BALT): a single centre experience," Interv. Neuroradiol, vol. 17, no. 3, pp. 306-315, 2011.

[3] B. Kundu, R. Rajkhowa, S. C. Kundu, and X. Wang, "Silk fibroin biomaterials for tissue regenerations," Adv Drug Deliv Rev, vol. 65, no. 5, pp. 457-470, 2013.

[4] L. Meinel, R. Fajardo, S. Hofmann, R. Langer, J. Chen, B. Snyder, G. Vunjak-Novakovic, and D. L. Kaplan, "Silk implants for the healing of critical size bone defects," Bone, vol. 37, pp. 688-698, 2005.

[5] C. R. Yoo, I. S. Yeo, K. E. Park, J. H. Park, S. J. Lee, W. H. Park, B. M. Min, "Effect of chitin/silk fibroin nanofibrous bicomponent structures on interaction with human epidermal keratinocytes," Int. J. Biol. Macromol, vol. 42, pp. 324-334, 2008.

[6] I. S. Yeo, J. E. Oh, L. Jeong, T. S. Lee, S. J. Lee, W. H. Park, B. M. Min, "Collagen-based biomimetic nanofibrous scaffolds: Preparation and characterization of collagen/silk fibroin bicomponent nanofibrous structures," Biomacromolecules, vol. 9, pp. 1106-1116, 2008.

[7] Y. Yang, F. Ding, J. Wu, W. Hu, W. Liu, J. Liu, and X. Gu, "Development and evaluation of silk fibroin-based nerve grafts used for peripheral nerve regeneration," Biomaterials, vol. 28, pp. 5526-5535, 2007.

[8] C. Y. Wang, K. H. Zhang, C. Y. Fan, X. M. Mo, H. J. Ruan, and F. F. Li, "Aligned natural -synthetic polyblend nanofibers for peripheral nerve regeneration," Acta Biomater, vol. 7, pp. 634-643, 2011.

[9] S. S. Silva, A. Motta, M. R. T. Rodrigues, A. F. M. Pinheiro, M. E. Gomes, J. O. F. Mano, R. L. Reis, and C. Migliaresi, "Novel Genipin-cross-linked chitosan/silk fibroin sponges for cartilage engineering strategies," Biomacromolecules, vol. 9, pp. 2764-2774, 2008.

[10] B. B. Mandal and S. C. Kundu, "Calcium alginate beads embedded in silk fibroin as 3D dual drug releasing scaffolds," Biomaterial, vol. 30, no. 28, pp. 5170-5177, 2009.

[11] X. Wang, T. Yucel, Q. Lu, X. Hu, and D. L. Kaplan, "Silk nanospheres and microspheres from silk/pva blend films for drug delivery," Biomaterials, vol. 31, no. 6, pp. 1025-1035, 2010.

[12] S. Hofmann, C. T. Foo, F. Rossetti, M. Textor, G. Vunjak-Novakovic, D. L. Kaplan, H. P. Merkle, and L. Meinel, "Silk fibroin as an organic polymer for controlled drug delivery," J. Control Release, vol. 111, pp. 217-227, 2006.

[13] X. Wang, E. Wenk, A. Matsumoto, L. Meinel, C. Li, D. L. Kaplan, "Silk microspheres for encapsulation and controlled release," J. Control Release, vol. 117, pp. 360-370, 2007.

[14] M. K. Cowman and S. Matsuoka, "Experimental approaches to hyaluronan structure," Carbohyd Res, vol. 340, no. 5, pp. 791-809, 2005.

[15] J. Necas, L. Bartosikova, P. Brauner, and J. Kolar, "Hyaluronic acid (hyaluronan): A review," Veterinarni Medicina, vol. 53, no. 8, pp. 397-411, 2008.

[16] M. N. Collins and C. Birkinshaw, "Hyaluronic acid based scaffolds for tissue engineering-A review," Carbobyd Polym, vol. 92, no. 2, pp. 1262-1279, 2013.

[17] C. E. Schanté, G. Zuber, C. Herlin, and T. F. Vandamme, "Chemical modifications of hyaluronic acid for the synthesis of derivatives for a broad range of biomedical applications," Carbohyd Polym, vol. 85, no. 3, pp. 469-489, 2011. 
[18] L. Lapč́k, L. Lapcik, S. De Smedt, J. Demeester, and P. Chabrecek, "Hyaluronan preparation, structure, properties, and applications," Chemical Reviews, vol. 98, no. 8, pp. 2663-2668, 1998.

[19] L. Han, Y. Zhao, L. Yin, R. Li, Y. Liang, H. Huang, S. Pan, C. Wu, and M. Feng, "Insulin-loaded pHsensitive hyaluronic acid nanoparticles enhance transcellular deliver," AAPS Pharm Sci Tech, vol. 13, no. 3, pp. 836-845, 2012.

[20] M. Manasa, V. Sridevi, M. V. V. Chandana Lakshmi and J .Dedeepya, "A Review on Hyaluronic Acid, "Int.J .Res .Chem .Environ, vol. 2, no. 4, pp. 6-11, 2012.

[21] I. Chattopadhyay, K. Biswas, U. Bandyopadhyay, K. B. Ranajit, "Turmeric and curcumin: Biological actions and medicinal applications," Curr Sci, vol. 87, no. 1, pp. 44-53, 2004.

[22] G. K. Jayaprakasha, L. Jaganmohan Rao, and K. K. Sakariah, "Antioxidant activities of curcumin, demethoxycurcumin and bisdemethoxycurcumin," Food Chem, vol. 98, no. 4, pp. 720-724, 2006.

[23] M. Shakibaei, T. John, G. Schulze-Tanzil, I. Lehmann, and A. Mobasheri, "Suppression of NF kappaB activation by curcumin leads to inhibition of expression of cyclo-oxygenase-2 and matrix metalloproteinase-9 in human articular chondrocytes: Implications for the treatment of osteoarthritis," Biochem Pharmacol, vol. 73, no. 9, pp. 1434-1445, 2007.

[24] Y. Henrotin, A. L. Clutterbuck, D. Allaway, E. M. Lodwig, P. Harris, M. Mathy-Hartert, M. Shakibaei, and A. Mobasheri, "Biological actions of curcumin on articular chondrocytes," Osteoarthr Cartilage, vol. 18, no. 2, pp. 141-149, 2010.

[25] Y. Henrotin, F. Priem, and A. Mobasheri, "Curcumin: A new paradigm and therapeutic opportunity for the treatment of osteoarthritis: curcumin for osteoarthritis management," Springer Plus, vol. 2, no. 56, pp. 1-9, 2013.

[26] B. Joe and R. Lokesh Belur, "Prophylactic and therapeutic effects of n-3 polyunsaturated capsaicin, and curcumin on adjuvant induced arthritis in rat," Nitritional Biochemistry, vol. 8, pp. 397-407, 1997.

[27] W. M. Weber, L. A. Hunsaker, S. F. Abcouwer, L. M. Deck, and D. L. Vander Jagt, "Anti-oxidant activities of curcumin and related enones," Bioorg Med Chem, vol. 13, no. 11, pp. 3811-3820, 2005.

[28] Z. M. Shao, Z. Z. Shen, C. H. Liu, M. R. Sartippour, V. L. Go, D. Heber, and M. Nguyen, "Curcumin exerts multiple suppressive effects on human breast carcinoma cells," Int J Cancer, vol. 98, no. 2, pp. 234-240, 2002.

[29] A. Duvoix, R .Blasius, S. Delhalle, M. Schnekenburger, F. Morceau, E. Henry, M. Dicato, and M. Diederich, "Chemopreventive and therapeutic effects of curcumin," Cancer Lett, vol. 223, no. 2, pp. 181-190, 2005.

[30] N. R. Jana, P. Dikshit, A. Goswami, and N. Nukina, "Inhibition of proteasomal function by curcumin induces apoptosis through mitochondrial pathway," J Biol Chem, vol. 279, no. 12, pp. 11680-11685, 2004.

[31] A. Shehzad, F. Wahid, and Y. S. Lee, "Curcumin in cancer chemoprevention: Molecular targets, pharmacokinetics, bioavailability, and clinical trials," Arch Pharm (Weinheim), vol. 343, no. 9, pp. 489499, 2010.

[32] R. Wilken, M. S. Veena, M. B. Wang, and E. S. Srivatsan, "Curcumin: A review of anti-cancer properties and therapeutic activity in head and neck squamous cell carcinoma," Mol Cancer, pp. 10-12, 2011.

[33] J. Ravindran, S. Prasad, and B. B. Aggarwal, "Curcumin and cancer cells :how many ways can curry kill tumor cells selectivel," AAPS J, vol. 11, no. 3, pp. 495-510, 2009.

[34] R. K. Das, N. Kasoju, and U. Bora, "Encapsulation of curcumin in alginatechitosan-pluronic composite nanoparticles for delivery to cancer cells," Nanomedicine, vol. 6, no. 1, pp. 153-160, 2010.

[35] C. Mohanty and S. K. Sahoo, "The in vitro stability and in vivo pharmacokinetics of curcumin prepared as an aqueous nanoparticulate formulation," Biomaterials, vol. 31, no. 25, pp. 6597-6611, 2010.

[36] K. Lerdchai, J. Kitsongsermthon, J. Ratanavaraporn, S. Kanokpanont, and S. Damrongsakkul, "Thai silk fibroin/gelatin sponges for the dual controlled release of curcumin and docosahexaenoic acid for anticancer treatment," J. Pharm Sci, vol. 105, no. 1, pp. 221-230, 2016.

[37] J. Ratanavaraporn, S. Kanokpanont, and S. Damrongsakkul, "The development of injectable gelatin/silk fibroin microspheres for the dual delivery of curcumin and piperine," J. Mater Sci Mater Med, vol. 25, no. 2, pp. 401-410, 2014.

[38] U. J. Kim, J. Park, H. J. Kim, M. Wada, and D. L. Kaplan, "Three-dimensional aqueous-derived biomaterial scaffolds from silk fibroin,” Biomaterials, vol. 26, no. 15, pp. 2775-2785, 2005. 
[39] R. L. Cleland and J. L. Wang, "Ionic polysaccharides III. Dilute solution properties of hyaluronic acid fractions," Biopolymers, vol. 9, pp. 799-810, 1970.

[40] B. Gunnar, "Determination of hexosamines according to Elson and Morgan," Acta Chemica Scandinavica, vol. 2, pp. 467-473, 1948.

[41] T. Takahashi, M. Ikegami-Kawai, R. Okuda, and K. Suzuki, "A fluorimetric Morgan-Elson assay method for hyaluronidase activity," Analytical Biochemistry, vol. 322, no. 2, pp. 257-263, 2003.

[42] K. Shahani and J. Panyam, "Highly loaded, sustained-release microparticles of curcumin for chemoprevention," J. Pharm Sci, vol. 100, no. 7, pp. 2599-2609, 2011.

[43] M. Okhawila, R. Rangkupan, S. Kanokpanont, and S. Damrongsakkul, "Preparation of Thai silk fibroin/gelatin electrospun fiber mats for controlled release applications," Int J Biol Macromol, vol. 46, no. 5, pp. 544-440, 2010.

[44] A. K. Dash and G. C. Cudworth, "Therapeutic applications of implantable drug delivery systems," J. Pharmacol Toxicol Methods, vol. 40, no. 1, pp. 1-12, 1998.

[45] P. L. Ritger and N. A. Peppas, "A simple equation for description of solute release II. Fickian and anomalous release from swellable devices," J. Control Release, vol. 5, pp. 37-42, 1987.

[46] J. Siepmann and N. A. Peppas, "Modeling of drug release from delivery systems based on hydroxypropyl methylcellulose (HPMC)," Adv Drug Deliver Rev, vol. 48, pp. 139-157, 2001.

[47] D. Y. Park, B. H. Min, D. W. Kim, B. R. Song, M. Kim, and Y. J. Kim, "Polyethylene wear particles play a role in development of osteoarthritis via detrimental effects on cartilage, meniscus, and synovium," Osteoarthritis and Cartilage, vol. 21, pp. 2021-2029, 2013.

[48] E. Rajalakshmanan, C. C. Chang, S. C. Wu, G. J. Wang, J. K. Chang, and M. L. Ho, "Sustained release of PTH(1-34) from PLGA microspheres suppresses osteoarthritis progression in rats," Acta Biomaterialia, vol. 8, pp. 2254-2262, 2012.

[49] A. D. John, Lange's Handbook of Chemistry, 15th ed. Knoxville, Tennessee: Chemistry, 1956.

[50] M. Li, M. Ogisob, and N. Minourab, "Enzymatic degradation behavior of porous silk fibroin sheets," Biomaterials, vol. 24, no. 2, pp. 357-365, 2003.

[51] C. Wongnarat and P. Srihanam, "Degradation behaviors of Thai bombyx mori silk fibroins exposure to protease enzymes, "Engineering, vol. 5, no. 1, pp. 61-66, 2013.

[52] Y. Y. Jo, H. Y. Kweon, Y. Koh, H. D. Kang, J. H. Yeo, et al., "Characteristics and Degradability of Silk Scaffold Fabricated by Press Method, Tissue Eng Regen Med, vol. 8, no. 1, pp. 42-46, 2011.

[53] N. Kasoju and U. Bora, "Silk fibroin in tissue engineering," Adv. Healthc. Mater, vol. 1,no. 4, pp. 393412, 2012

[54] Y. Lian, J.C. Zhan, K. H. Zhang, and X. M. Mo, "Fabrication and characterization of curcumin loaded silk fibroin/P)LLA-CL (nanofibrous scaffold)," Frontiers of Materials Science, vol. 8, no. 4, pp. 354-362, 2014. 